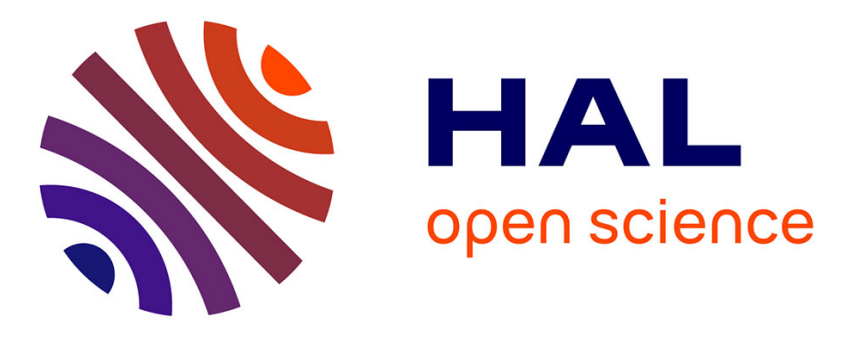

\title{
Practical Analysis of RSA Countermeasures Against Side-Channel Electromagnetic Attacks
}

\author{
Guilherme Perin, Laurent Imbert, Lionel Torres, Philippe Maurine
}

\section{To cite this version:}

Guilherme Perin, Laurent Imbert, Lionel Torres, Philippe Maurine. Practical Analysis of RSA Countermeasures Against Side-Channel Electromagnetic Attacks. CARDIS: Smart Card Research and Advanced Applications, Nov 2013, Berlin, Germany. pp.200-215, 10.1007/978-3-319-08302-5_14 . lirmm-01096070

\section{HAL Id: lirmm-01096070 https://hal-lirmm.ccsd.cnrs.fr/lirmm-01096070}

Submitted on 16 Dec 2014

HAL is a multi-disciplinary open access archive for the deposit and dissemination of scientific research documents, whether they are published or not. The documents may come from teaching and research institutions in France or abroad, or from public or private research centers.
L'archive ouverte pluridisciplinaire HAL, est destinée au dépôt et à la diffusion de documents scientifiques de niveau recherche, publiés ou non, émanant des établissements d'enseignement et de recherche français ou étrangers, des laboratoires publics ou privés. 
Practical Analysis of RSA

\section{Countermeasures Against Side-Channel Electromagnetic Attacks}

Guilherme Perin, Laurent Imbert, Lionel Torres and Philippe Maurine

November 28th, 2013

CARDIS 2013 - 12th Smart Card Research and Advanced Application Conference 


\section{Motivation}

- RSA is a continuing subject of many side-channel attacks

- Is there a combination of countermeasures which provides sufficient protection against most advanced side-channel attacks?

- Simple and Collisions-based Attacks

- Differential and Correlation Analyses

- Single Execution Attacks on Exponentiations

- Different levels of countermeasures 


\section{Agenda}

- Countermeasures

- RNS-based RSA

- The Proposed Hardware

- Robustness Against Electromagnetic Analysis:

- Collision-based attacks

- Correlation Analyses

- EM Analysis vs Hardware Countermeasures 


\section{RSA: Countermeasures}

\section{Algorithmic: Blinded Exponentiation}

$$
\begin{array}{r}
N=p \times q \\
\phi(N)=(p-1)(q-1) \\
c=m^{e} \bmod N
\end{array}
$$

$e r=e+r . \phi(N)$

$A_{0}=1+r_{1} . n \bmod r_{2} . n$

$A_{1}=m+r_{1} \cdot n \bmod r_{2} \cdot n$

for $\quad i=t-1: 0$

$A_{\overline{e r}}=A_{0} \cdot A_{1} \bmod N$

$A_{e r_{i}}=A_{e r_{i}} \cdot A_{e r_{i}} \bmod N$
Exponent Blinding

Additive Message Blinding

Regular Exponentiation:

Montgomery Ladder

end for 


\section{RSA: Countermeasures}

2. Hardware

- Minimize the Signal-to-Noise Ratio (SNR)

- Variable location (localized EM analyses)

- Clock jitter

- Dummy cycles

- Frequency dividers

Single Execution (Trace) Attacks on Exponentiation:

- Horizontal Attacks

- Supervised and Unsupervised Template Attacks 


\section{RSA: Countermeasures}

\section{Arithmetic: The Leak Resistant Arithmetic*}

- LRA is a derivative of RNS arithmetic for PKC algorithms;

- RNS is a fast, parallel and natural msg blinding arithmetic;

- Immune to collision, differential and (vertical/horizontal) correlation attacks.

- $C_{k}^{2 k} \approx 2^{2 k} / \sqrt{\pi k}$ different representations ( $k=$ number of moduli).

All variables are randomized during the exponentiation:

- Moduli could be recovered during the Radix to RNS Conversion

- For 32 moduli: Prob[moduli guessed $=$ moduli hard ware $]=1.65 .10^{-9}$

- Preliminar conclusion: vulnerabilites will be only related to RAM and CPU executions (conditional tests, addressing, etc.)

* J.-C. Bajard, L. Imbert, P.-Y. Liardet, and Y. Teglia, "Leak resistant arithmetic," in CHES'04, ser. LNCS, vol. 


\section{Agenda}

- Countermeasures

- RNS-based RSA

- The Proposed Hardware

- Robustness Against Electromagnetic Analysis:

- Collision-based attacks

- Correlation Analyses

- EM Analysis vs Hardware Countermeasures 


\section{Residue Number System}

A integer $\boldsymbol{X}$ is represented according to a base $\mathcal{B}=\left(b_{1}, b_{2}, \ldots, b_{n}\right)$ of relatively prime integers (moduli). Then:

$$
\langle X\rangle_{\mathcal{B}}=\left(x_{1}, x_{2}, \ldots, x_{k}\right)
$$

where $\boldsymbol{x}_{\boldsymbol{i}}=\boldsymbol{X} \bmod \boldsymbol{b}_{\boldsymbol{i}}$. Then, operations,,+- . are performed modulo $b_{i}$ :

$$
\begin{array}{r}
x_{i}+y_{i} \bmod b_{i} \\
x_{i}-y_{i} \bmod b_{i} \\
x_{i} \cdot y_{i} \bmod b_{i}
\end{array}
$$

Notation: $|X|_{b_{i}}=X \bmod b_{i}$ 


\section{RNS Montgomery Ladder}

Data: $x$ in $\mathcal{A} \cup \mathcal{B}$, where $\mathcal{A}=\left(a_{1}, a_{2}, \ldots, a_{k}\right), \mathcal{B}=\left(b_{1}, b_{2}, \ldots, b_{k}\right), A=\prod_{i=1}^{k} a_{i}$,

$$
B=\prod_{i=1}^{k} b_{i}, \operatorname{gcd}(A, B)=1, \operatorname{gcd}(B, N)=1 \text { and } e=\left(e_{n}-1 \ldots e_{1} e_{0}\right)_{2} \text {. }
$$

Result: $z=x^{e} \bmod N$ in $\mathcal{A} \cup \mathcal{B}$

Pre-Computations: $|A B \bmod N|_{\mathcal{A} \cup \mathcal{B}}$

$$
\begin{aligned}
& A_{0}=M M(1, A B \bmod N, N, \mathcal{A}, \mathcal{B}) \quad(\text { in } \mathcal{A} \cup \mathcal{B}) \\
& A_{1}=M M(x, A B \bmod N, N, \mathcal{A}, \mathcal{B}) \quad(\text { in } \mathcal{A} \cup \mathcal{B}) \\
& \text { for } i=n-1 \text { to } 0 \text { do } \\
& A_{\overline{e_{i}}}=M M\left(A_{\overline{e_{i}}}, A_{e_{i}}, N, \mathcal{B}, \mathcal{A}\right) \quad(\text { in } \mathcal{A} \cup \mathcal{B}) \\
& A_{e_{i}}=M M\left(A_{e_{i}}, A_{e_{i}}, N, \mathcal{B}, \mathcal{A}\right) \quad(\text { in } \mathcal{A} \cup \mathcal{B})
\end{aligned}
$$

end

$A_{0}=M M\left(A_{0}, 1, N, \mathcal{B}, \mathcal{A}\right) \quad($ in $\mathcal{A} \cup \mathcal{B})$

Transform the input data $(1, x)$ into the Montgomery domain by inverting $\mathcal{A}$ and $\mathcal{B}$ In the two calls of MM:

$>1 \cdot A B \cdot A^{-1} \bmod N=1 \cdot A^{2} B \bmod N=B \bmod N$

$>x \cdot A B \cdot A^{-1} \bmod N=x \cdot A^{2} B \bmod N=x \cdot B \bmod N$ 


\section{Montgomery Multiplication}

Classical arithmetic: (Montgomery Constant $\mathbf{R}=\mathbf{2}^{\mathrm{k}}, \mathrm{k}$ is the bitlength)

$$
\begin{aligned}
& q=x \cdot y \cdot\left(-N^{-1}\right) \bmod R \\
& s=\frac{x \cdot y+q \cdot N}{R} \quad \text { Return } \mathbf{x} \cdot \mathbf{y} \cdot \mathbf{R}^{-1} \bmod \mathbf{N}
\end{aligned}
$$

Residue Number System: (Montgomery Constant $B=\prod_{i=1}^{k} b_{i}$, $\mathrm{k}$ is the number of moduli in base $\mathcal{B}=\left(b_{1}, \ldots, b_{i}\right)$ )

$$
\begin{array}{l|c|l}
\text { Base } \mathcal{A} & \text { Base Extension } & \text { Base } \mathcal{B} \\
q_{\mathcal{A}} & \leftarrow & q_{\mathcal{B}}=x_{\mathcal{B}} \cdot y_{\mathcal{B}} \cdot\left|-N^{-1}\right|_{\mathcal{B}} \\
w_{\mathcal{A}}=\left(x_{\mathcal{A}} \cdot y_{\mathcal{A}}+q_{\mathcal{A}} \cdot N_{\mathcal{A}}\right) / B & \rightarrow & w_{\mathcal{B}}
\end{array}
$$

Return x.y. $\mathrm{B}^{-1} \bmod \mathbf{N}$ 


\section{RNS Montgomery Multiplication}

$s_{\mathcal{B}}=x_{\mathcal{B}} \cdot y_{\mathcal{B}}$

Fast Approximation Base Extension (CRT):

$s_{\mathcal{A}}=x_{\mathcal{A}} \cdot y_{\mathcal{A}}$

$q_{\mathcal{B}}=s_{\mathcal{B}} \cdot\left|-N^{-1}\right|_{\mathcal{B}}$

$$
X=\sum_{i=1}^{k} B_{i}\left|x_{i} B_{i}^{-1}\right| b_{i}-f . B \quad B_{i}=\frac{B}{b_{i}}
$$

$q_{\mathcal{A}} \leftarrow q_{\mathcal{B}}$

$|X|_{\mathcal{A}}=\left.\left.\left|\sum_{i=1}^{k} B_{i}\right| x_{i} B_{i}^{-1}\right|_{b_{i}}\right|_{a_{i}}-f .|B|_{a_{i}}$

$w_{\mathcal{A}}=\left(s_{\mathcal{A}}+q_{\mathcal{A}} \cdot N_{\mathcal{A}}\right) \cdot B^{-1}$

$w_{\mathcal{B}} \leftarrow w_{\mathcal{A}}$

$$
\begin{aligned}
& f=\left\lfloor\left(\sum_{i=1}^{k}\left|q \cdot B_{i}^{-1}\right|_{b_{i}}\right) / 2^{m}\right\rfloor \\
& q_{\mathcal{A}}=\left.\left.\left|\sum_{i=1}^{k}\right| q\right|_{b_{i}} \cdot B_{i}\right|_{\mathcal{A}}-|f . B|_{\mathcal{A}}
\end{aligned}
$$

BE2 $f=\left\lfloor\left(2^{m-1}+\sum_{i=1}^{k}\left|w \cdot A_{i}^{-1}\right|_{a_{i}}\right) / 2^{m}\right\rfloor$

$$
w_{\mathcal{B}}=\left.\left.\left|\sum_{i=1}^{k}\right| w\right|_{a_{i}} \cdot A_{i}\right|_{\mathcal{B}}-|f . A|_{\mathcal{B}}
$$




\section{RNS Montgomery Multiplication Improved Version [ ${ }^{*}$ ]}

$$
\begin{aligned}
& s_{\mathcal{B}}=x_{\mathcal{B}} \cdot y_{\mathcal{B}} \\
& s_{\mathcal{A}}=x_{\mathcal{A}} \cdot y_{\mathcal{A}} \\
& q_{\mathcal{B}}=\left|s_{\mathcal{B}} \cdot B_{i}^{-1} \cdot-N^{-1}\right|_{\mathcal{B}} \\
& f=\left\lfloor\left(\sum_{i=1}^{k}|q|_{b_{i}}\right) / 2^{m}\right\rfloor \\
& w_{\mathcal{A}}=s_{\mathcal{A}} \cdot B^{-1}+\left.\sum_{i=1}^{k}|q|_{b_{i}} \cdot B_{i} \cdot N \cdot B^{-1}\right|_{\mathcal{A}}-\left|f . B . N \cdot B^{-1}\right|_{\mathcal{A}} \\
& q_{\mathcal{A}}=\left|w \cdot A_{i}^{-1}\right|_{\mathcal{A}} \\
& f=\left\lfloor\left(2^{m-1}+\sum_{i=1}^{k}|q|_{a_{i}}\right) / 2^{m}\right\rfloor \\
& w_{\mathcal{B}}=\left.\left.\left|\sum_{i=1}^{k}\right| w\right|_{a_{i}} \cdot A_{i}\right|_{\mathcal{B}}-|f \cdot A|_{\mathcal{B}}
\end{aligned}
$$

\begin{tabular}{|l|l} 
RNS MM & $\begin{array}{l}\text { RNS MM } \\
\text { Improved }\end{array}$ \\
\hline $2 k^{2}+7 k$ & $2 k^{2}+5 k$
\end{tabular}




\section{Agenda}

- Countermeasures

- RNS-based RSA

- The Proposed Hardware

- Robustness Against Electromagnetic Analysis:

- Collision-based attacks

- Correlation Analyses

- EM Analysis vs Hardware Countermeasures 


\section{Proposed and Evaluated Hardware}

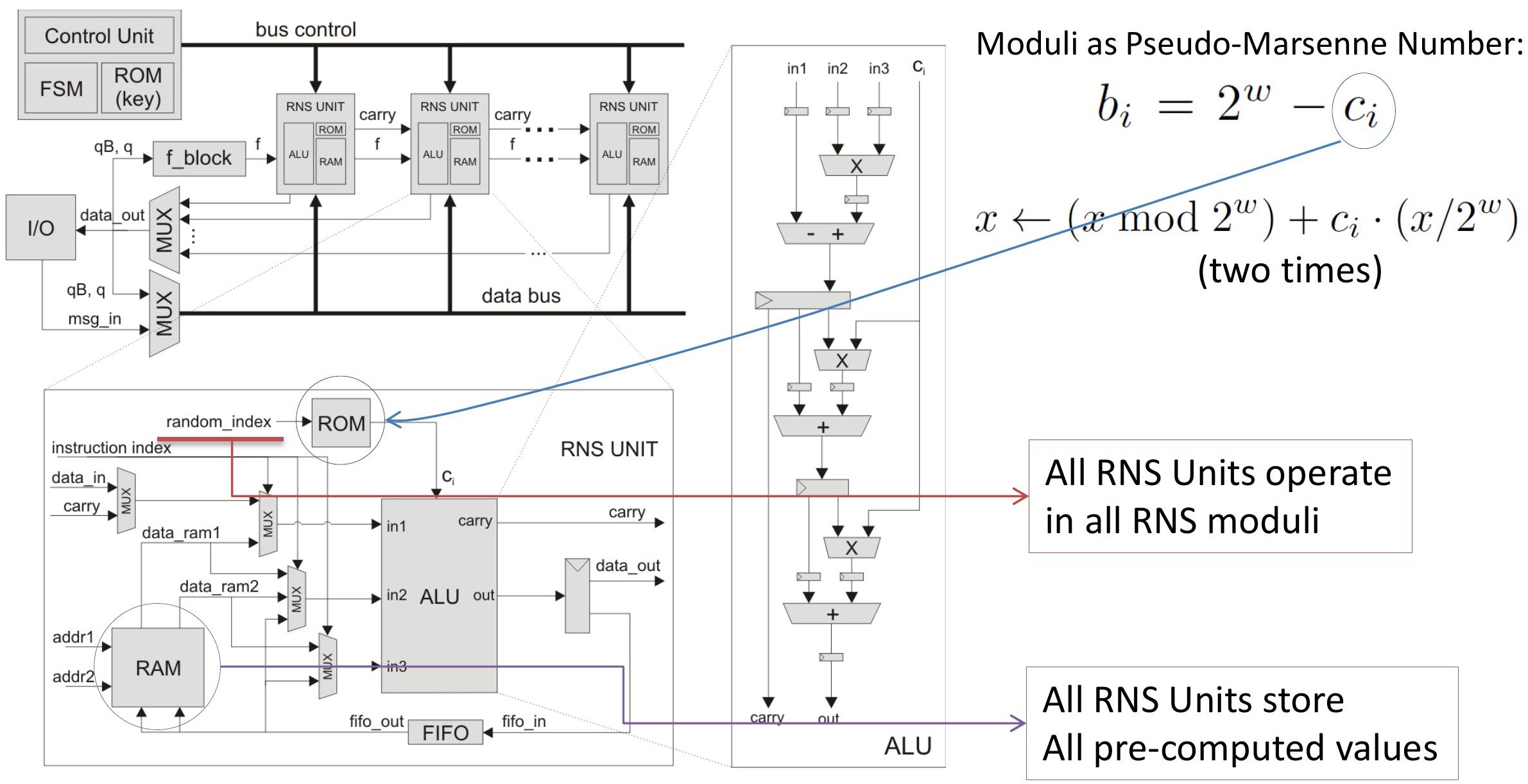

With Fixed Bases (32 moduli, 32 bits): pre-computations need 8.5 kB With Randomized Bases (32 moduli, 32 bits): pre-computations need 118 kB 


\section{LRA Precomputations}

- RNS Bases are randomized once before each exponentiation.

- Clock cycles (512 bits):

Fixed RNS Bases

\begin{tabular}{|c|c|c|}
\hline FC & EXPONENTIATION & $\mathrm{RC}$ \\
\hline 48 & 78210 & 685 \\
\hline
\end{tabular}

Randomized RNS Bases

\begin{tabular}{|c|c|c|c|}
\hline FC & LRA & EXPONENTIATION & RC \\
\hline 48 & 1060 & 78210 & 840
\end{tabular}

$\mathrm{FC}=$ Radix to RNS

Clock Cycles Overhead: 1\%

$\mathrm{RC}=\mathrm{RNS}$ to Radix

Memory Overhead: $92 \%$ 


\section{Agenda}

- Countermeasures

- RNS-based RSA

- The Proposed Hardware

- Robustness Against Electromagnetic Analysis:

- Collision-based attacks

- Correlation Analyses

- EM Analysis vs Hardware Countermeasures 


\section{Collision Attacks}

- Identify redundant operations by collecting two (averaged or not) traces for different chosen-message pairs:

$-\left(x, x^{2}\right)$ : Doubling Attack

- $(\mathrm{x},-\mathrm{x})$ : Yen's et al Attack

- $\left(x^{\alpha}, y^{\beta}\right):$ Homma's et al Attack

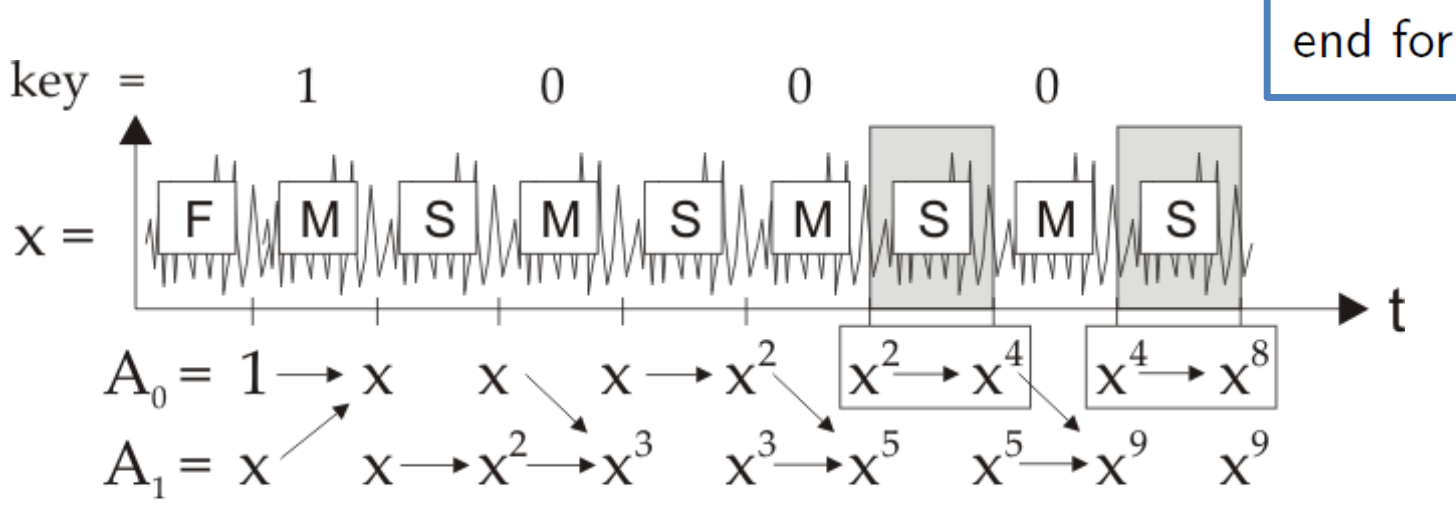

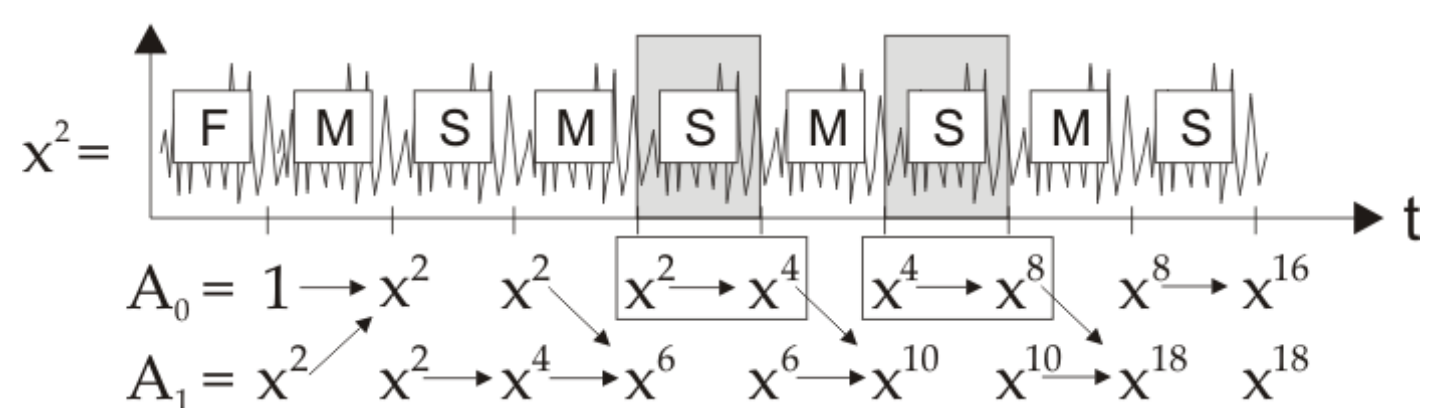

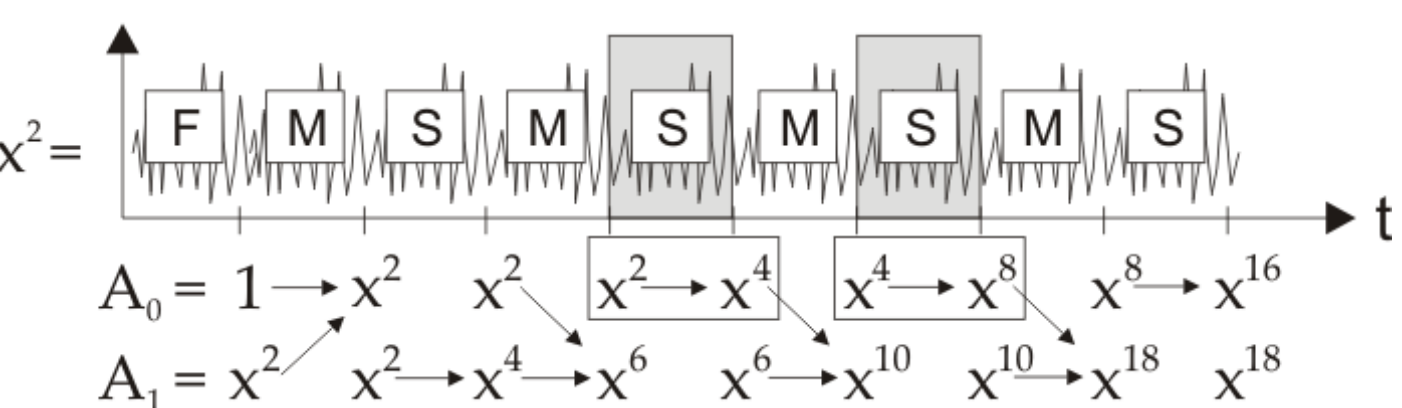

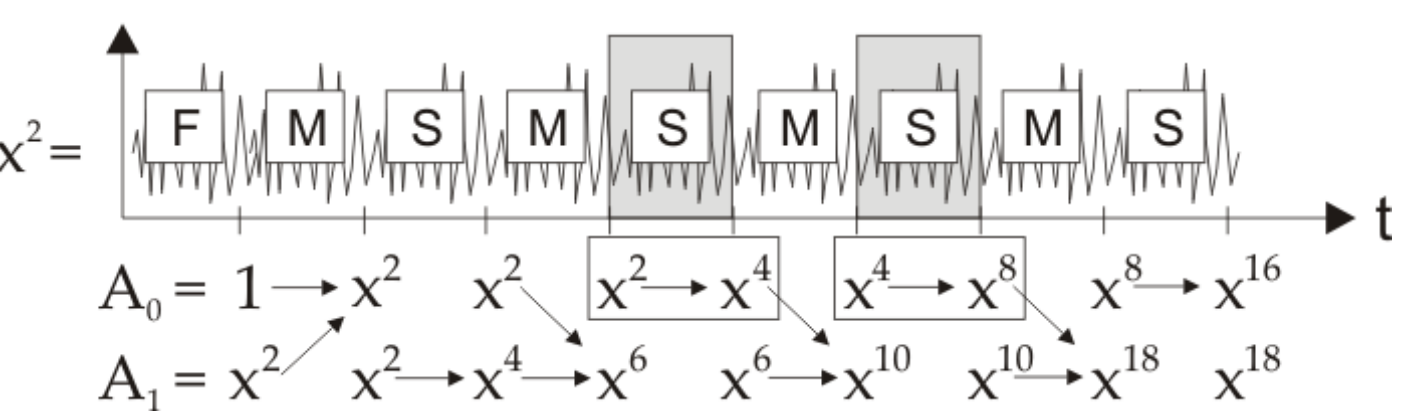

$$
\text { for } \begin{aligned}
i & =t-1: 0 \\
A_{\overline{e r_{i}}} & =A_{0} \cdot A_{1} \bmod N \\
A_{e r_{i}} & =A_{e r_{i}} \cdot A_{e r_{i}} \bmod N
\end{aligned}
$$




\section{LRA vs Collision Attacks}
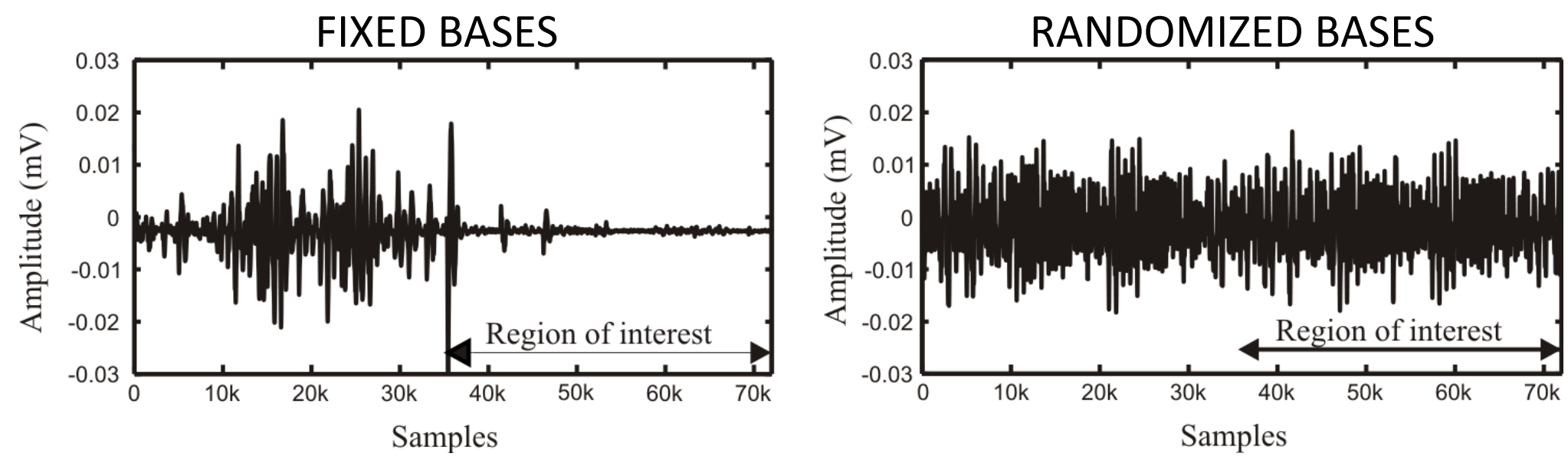

$\operatorname{EM}\left(T_{S}, x, e_{i}\right)=$ squaring EM trace at $e_{i}$ $\operatorname{EM}\left(T_{S}, x, e_{i-1}\right)=$ squaring $E M$ trace at $e_{i-1}$

$S N R=20 . \log _{10} \frac{P_{\text {signal }}}{P_{\text {noise }}}=$

$=20 . \log _{10} \frac{\sigma_{\left(E M\left(T_{S}, x, e_{i-1}\right)\right)}^{2}}{\sigma_{\left(E M\left(T_{S}, x, e_{i-1}\right)-E M\left(T_{S}, x^{2}, e_{i}\right)\right)}^{2}}$
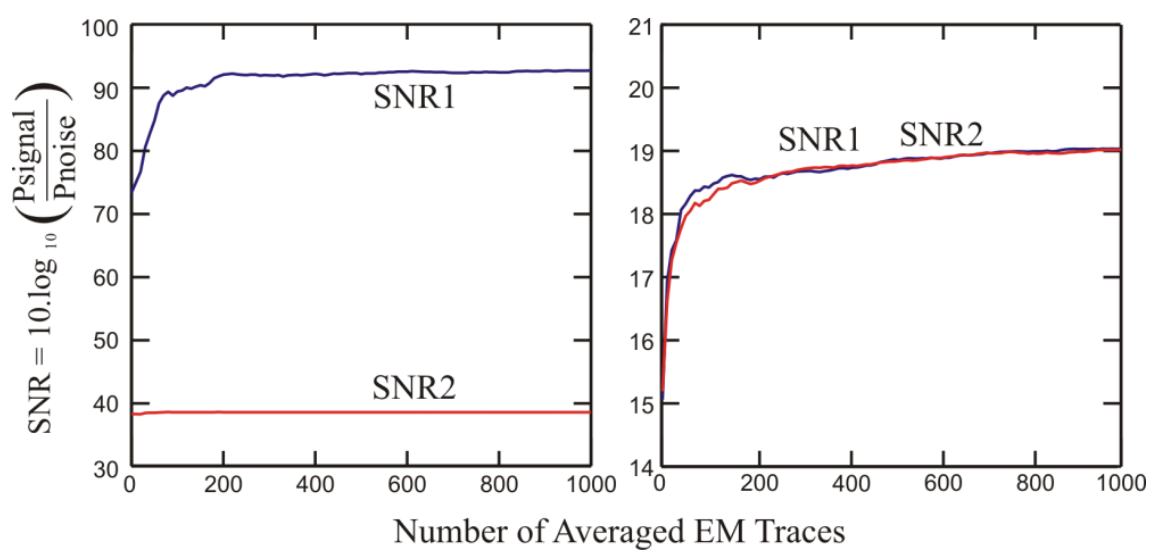


\section{Correlation Attacks}

$H W_{m}=$ Hamming Weight of a Data $m$

$t_{i}(j)=$ sample $j$ of a trace $i$

$$
\rho\left(H W_{m}, t_{i}(j)\right)=\frac{\operatorname{cov}\left(H W_{m}, t_{i}(j)\right)}{\sqrt{\operatorname{var}\left(H W_{m}\right) \operatorname{var}\left(t_{i}(j)\right)}}
$$

Vertical:

- Correlate HW x Trace

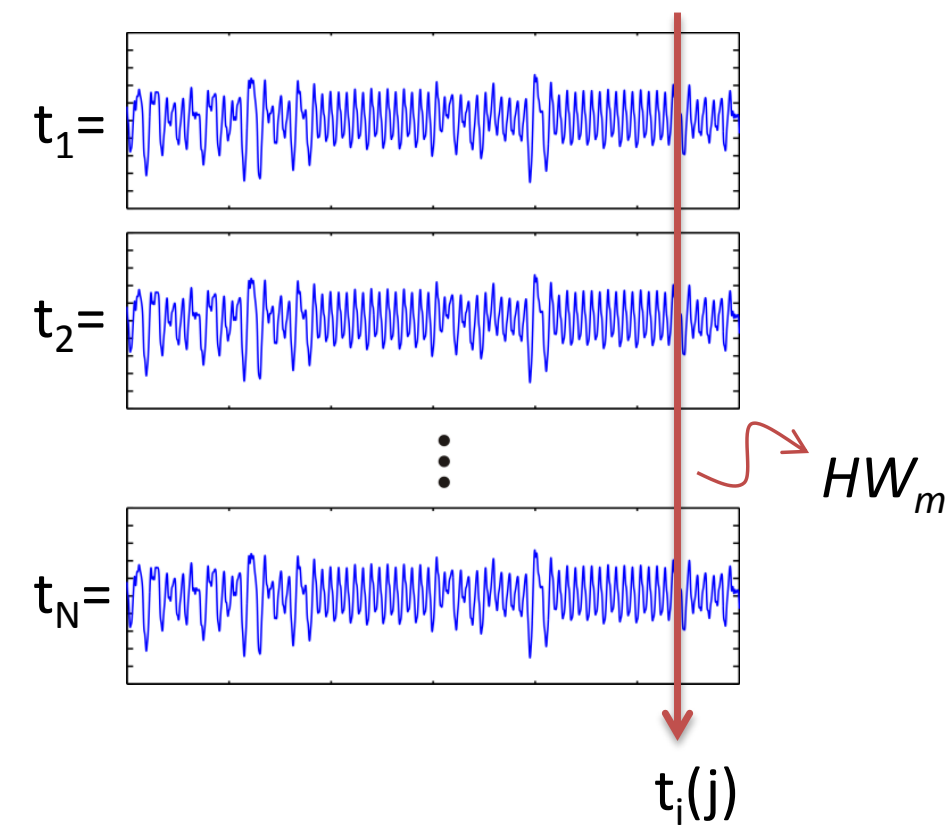

Horizontal (Immune to Exponent Blinding):

- Correlate HW x Trace

- Correlate Trace x Trace*

\section{A.m $\bmod N \quad$ A.A $\bmod N \quad$ A.m $\bmod N$}

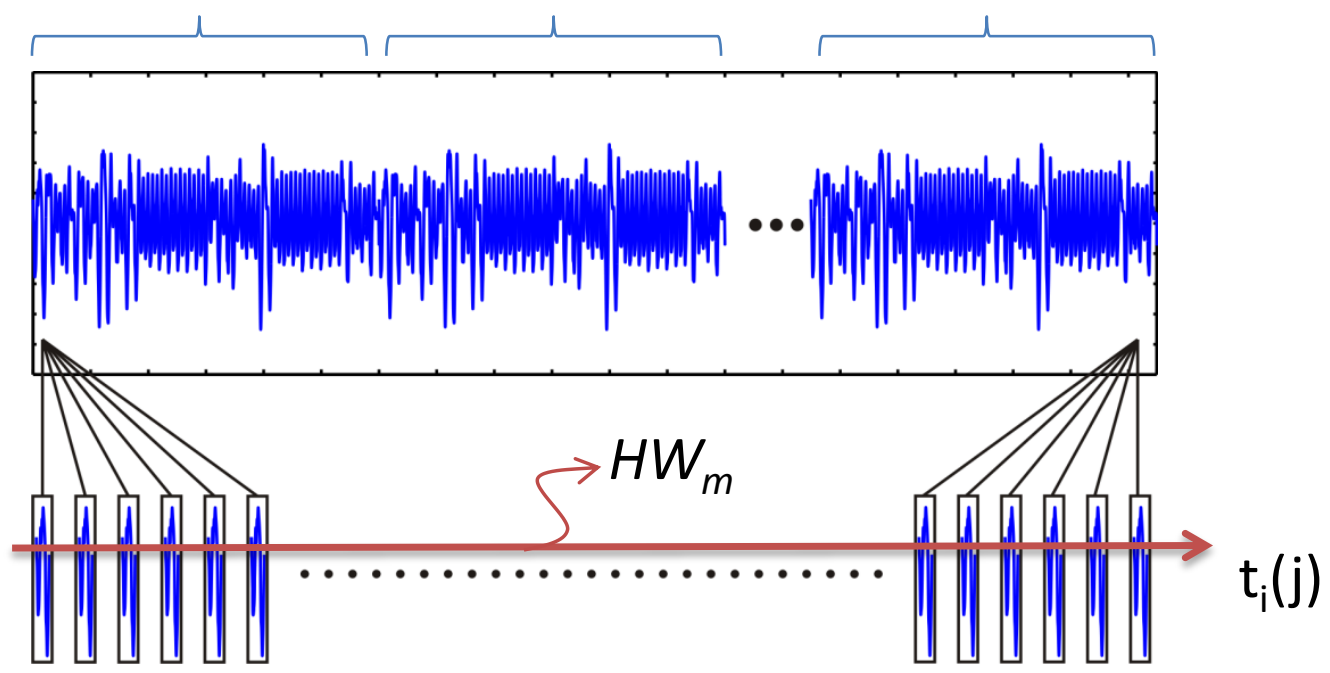

${ }^{*}$ C. Clavier, B. Feix, G. Gagnerot, C. Giraud, M. Rousselet and V. Verneuil, "ROSETTA for Single Trace Analysis," in 


\section{LRA vs Correlation Attacks}

Vertical:

FIXED BASES

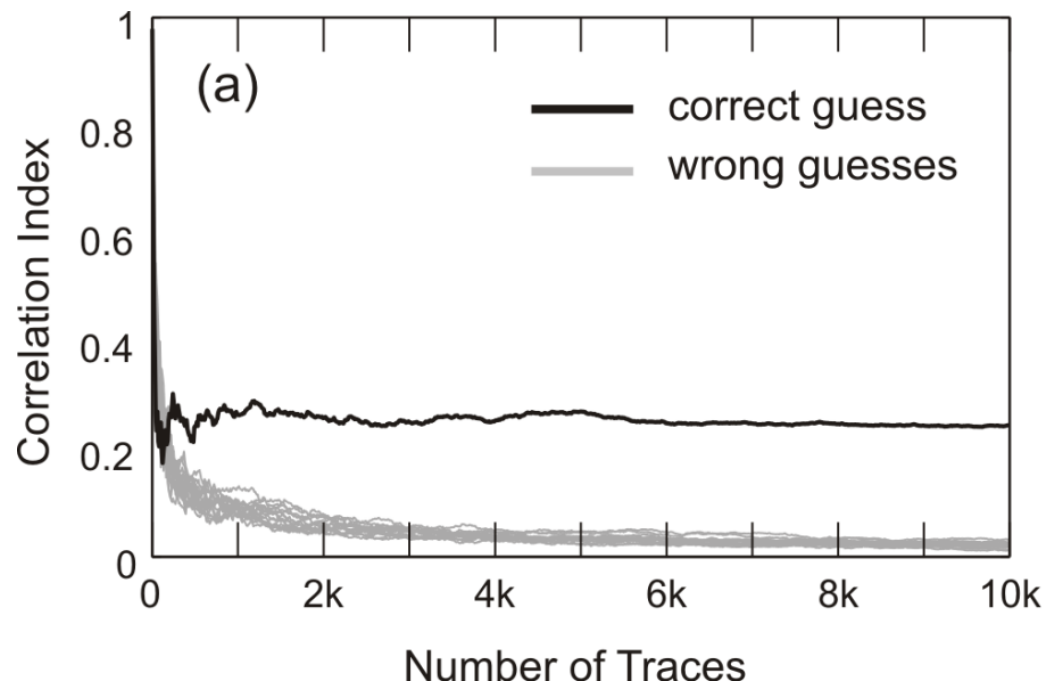

Horizontal: Proposed for Long-Integer Multiplications

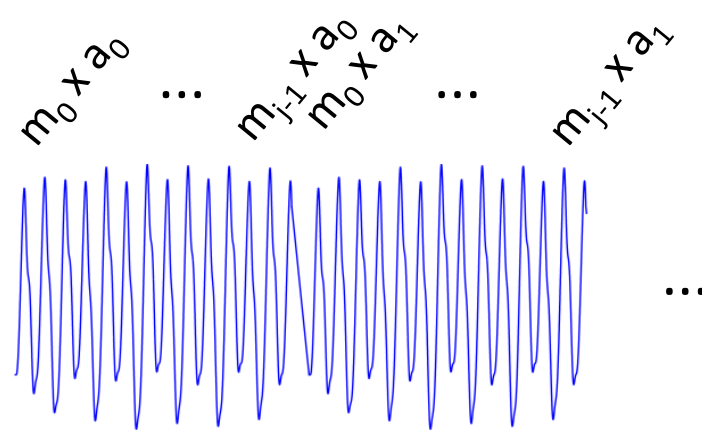

RANDOMIZED BASES

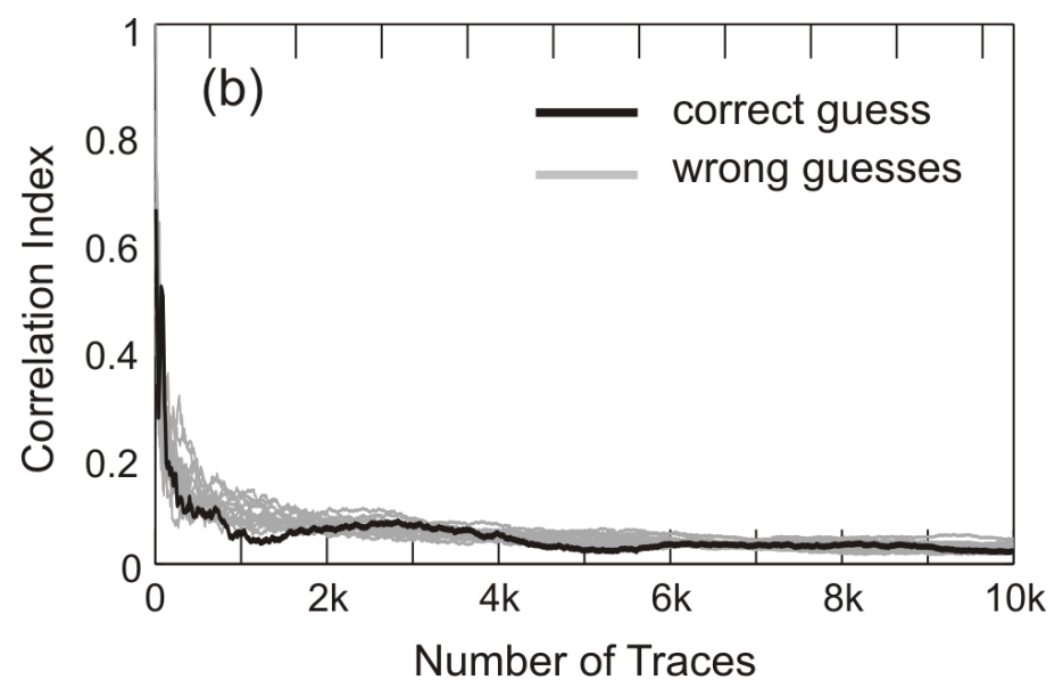

Horizontal: RNS Multiplications

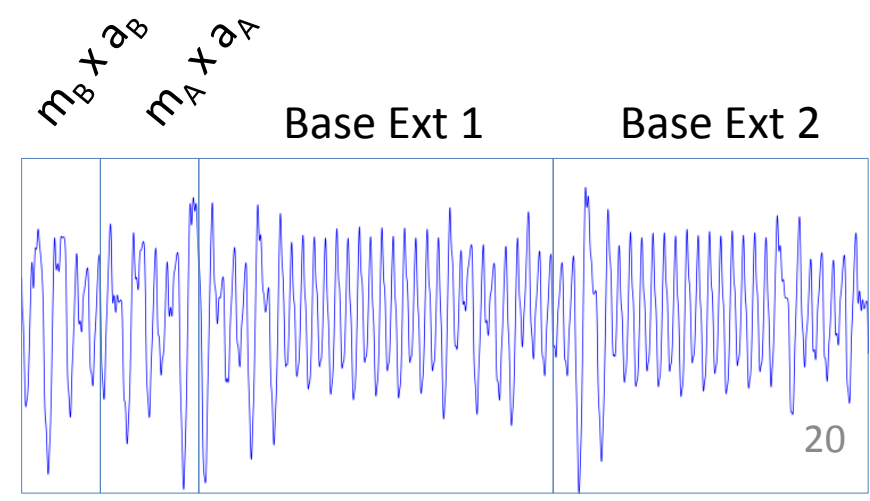




\section{Single Execution Attacks}

- Why? Exponentiation is randomized.

- Exponent: er $=e+r . \phi(N)$

- Message: Leak Resistant Arithmetic

- Which attacks?

- Horizontal attacks;

- Supervised, semi-supervised and unsupervised template attacks:

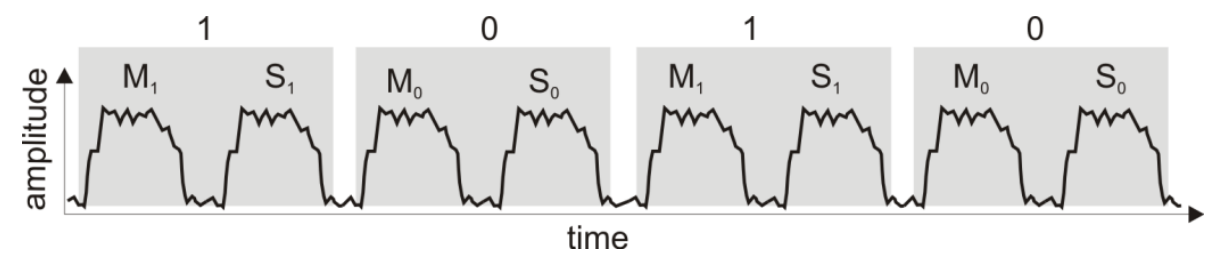

$\square$ Montgomery Ladder -> Find the means $(\mu)$ and std dev $(\sigma)$ of two classes:

- $\mathrm{N}\left(\mu_{(\mathrm{m} 0)}, \sigma_{(\mathrm{mo})}\right):$ mean and std dev of a multiplication when exponent bit is 0

- $\mathrm{N}\left(\mu_{(\mathrm{m} 1)}, \sigma_{(\mathrm{m} 1)}\right)$ : mean and std dev of a multiplication when exponent bit is 1

- $\mathrm{N}\left(\mu_{(\mathrm{s})}, \sigma_{(\mathrm{mo})}\right)$ : mean and std dev of a squaring when exponent bit is 0

- $\mathrm{N}\left(\mu_{(\mathrm{s} 1)}, \sigma_{(\mathrm{m} 1)}\right)$ : mean and std dev of a squaring when exponent bit is $\mathbf{1}$ 


\section{Single Execution Attacks on RNS Exponentiation}

RAM, CPU: exponent-dependent activities

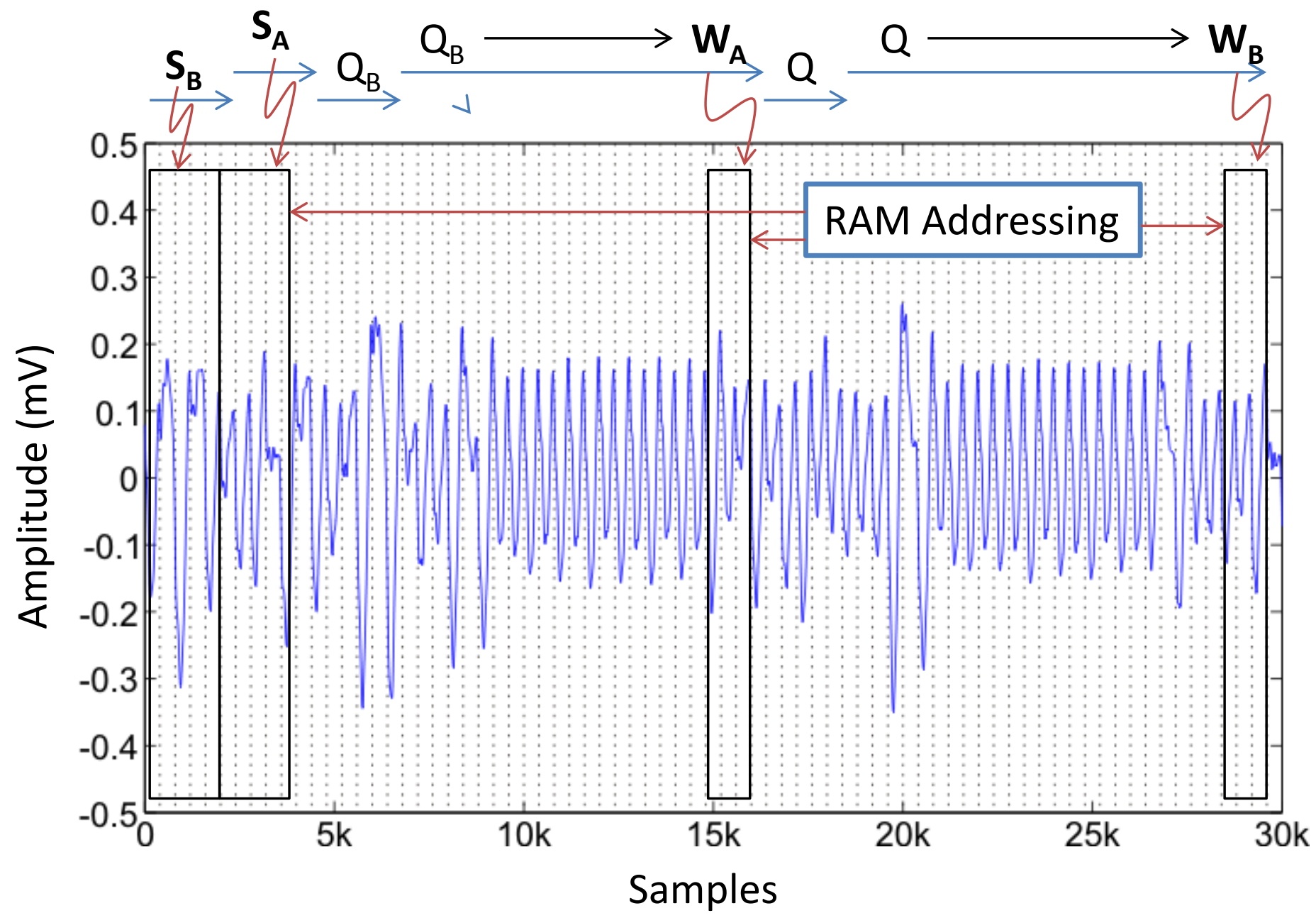




\section{What are the RAM leakages?}

- Fixed Exponent:

- Averaged EM traces: remove the data dependency

$$
\begin{aligned}
& \overline{m_{0}}=\frac{1}{N_{t r}} \sum_{i=1}^{N_{t r}} m_{i}(0) \\
& \overline{m_{1}}=\frac{1}{N_{t r}} \sum_{i=1}^{N_{t r}} m_{i}(1) \\
& \overline{s_{0}}=\frac{1}{N_{t r}} \sum_{i=1}^{N_{t r}} s_{i}(0) \\
& \overline{s_{1}}=\frac{1}{N_{t r}} \sum_{i=1}^{N_{t r}} s_{i}(1)
\end{aligned}
$$
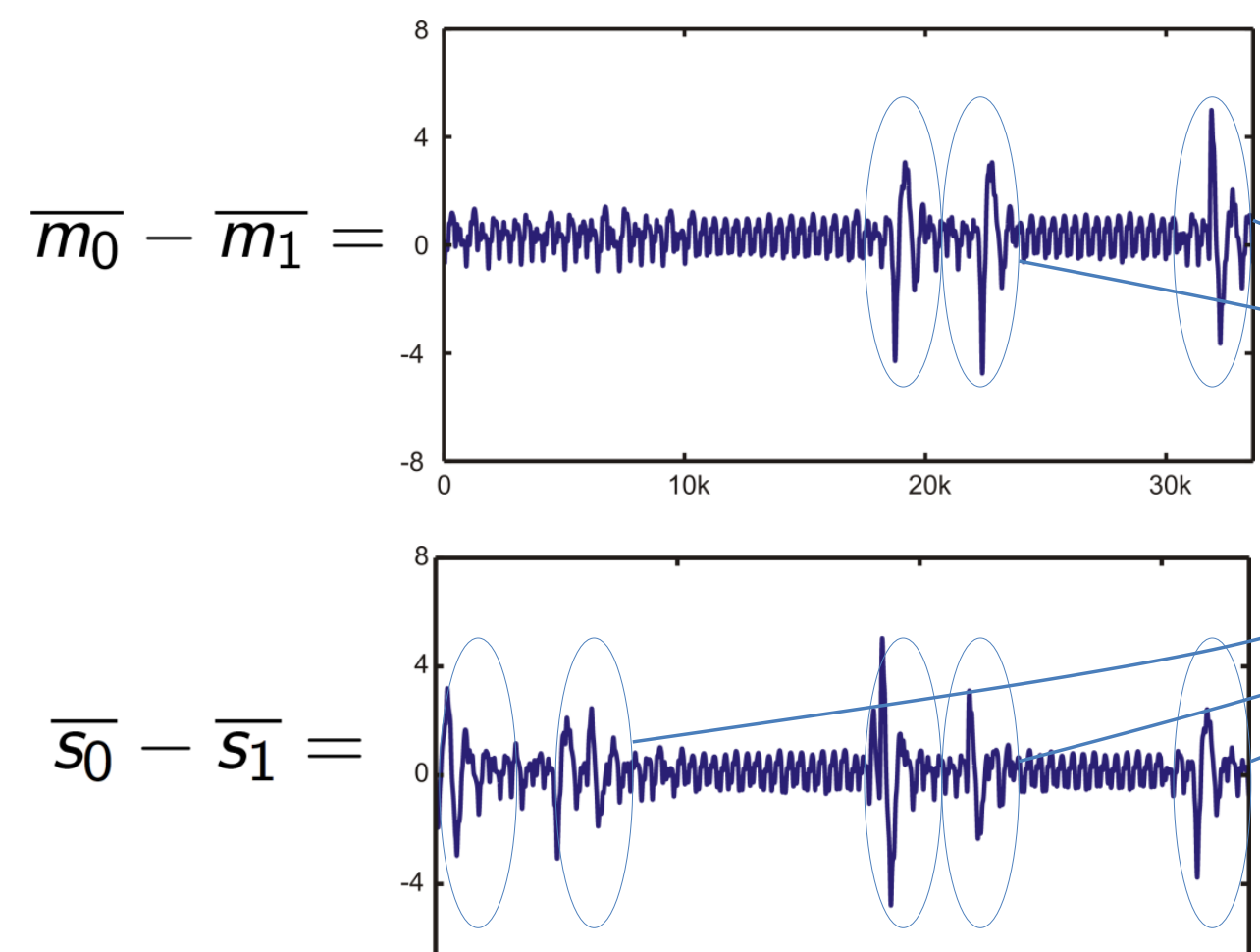

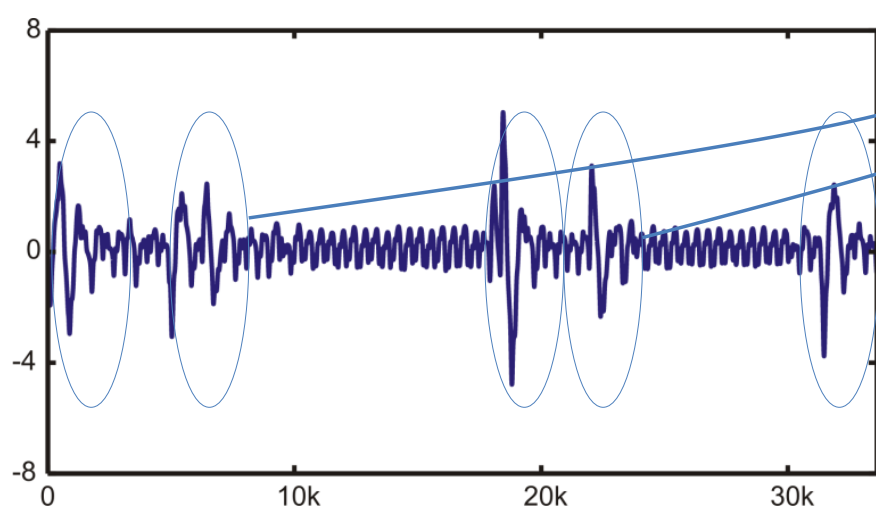

Conditional Tests RAM Addressing 


\section{RAM Addressing Randomization}

Intermediate results are never stored in same positions:

$$
\begin{aligned}
& \text { for } \begin{aligned}
i & =t-1: 0 \\
A_{\overline{e r_{i}}} & =A_{0} \cdot A_{1} \bmod N \\
A_{e r_{i}} & =A_{e r_{i}} \cdot A_{e r_{i}} \bmod N
\end{aligned} \\
& \text { end for }
\end{aligned}
$$

Multiplication(1):

$\mathrm{A} 0=\mathrm{A} 0 . \mathrm{A} 1 \bmod \mathrm{N}$

$\operatorname{read}(A 0, A 1)$

write(A0)
Squaring(1):

$\mathrm{A} 1=\mathrm{A} 1 . \mathrm{A} 1 \bmod \mathrm{N}$

$\operatorname{read}(A 1)$

write(A1)
Multiplication(0):

$\mathrm{A} 1=\mathrm{A} 0 . \mathrm{A} 1 \bmod \mathrm{N}$

$\operatorname{read}(A 1)$

write(A1)
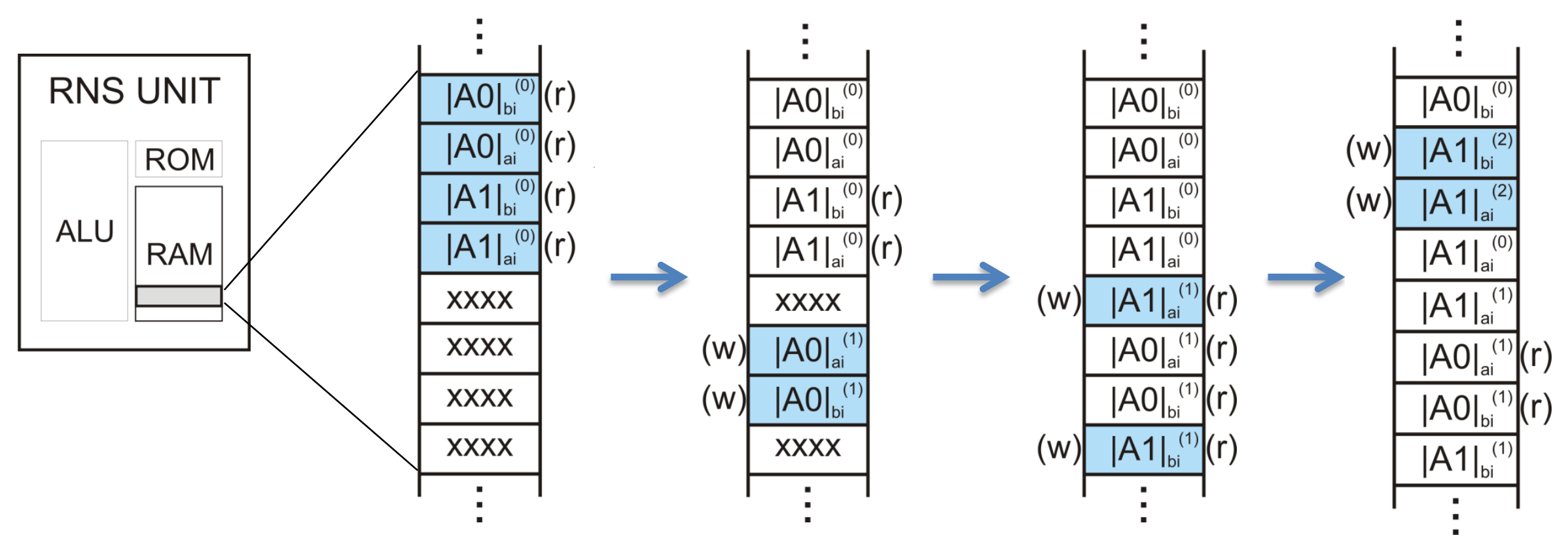


\section{RAM Addressing Randomization}

We took a fixed sample point $t_{i}$ representing the RAM addressing (writing):

Unprotected:
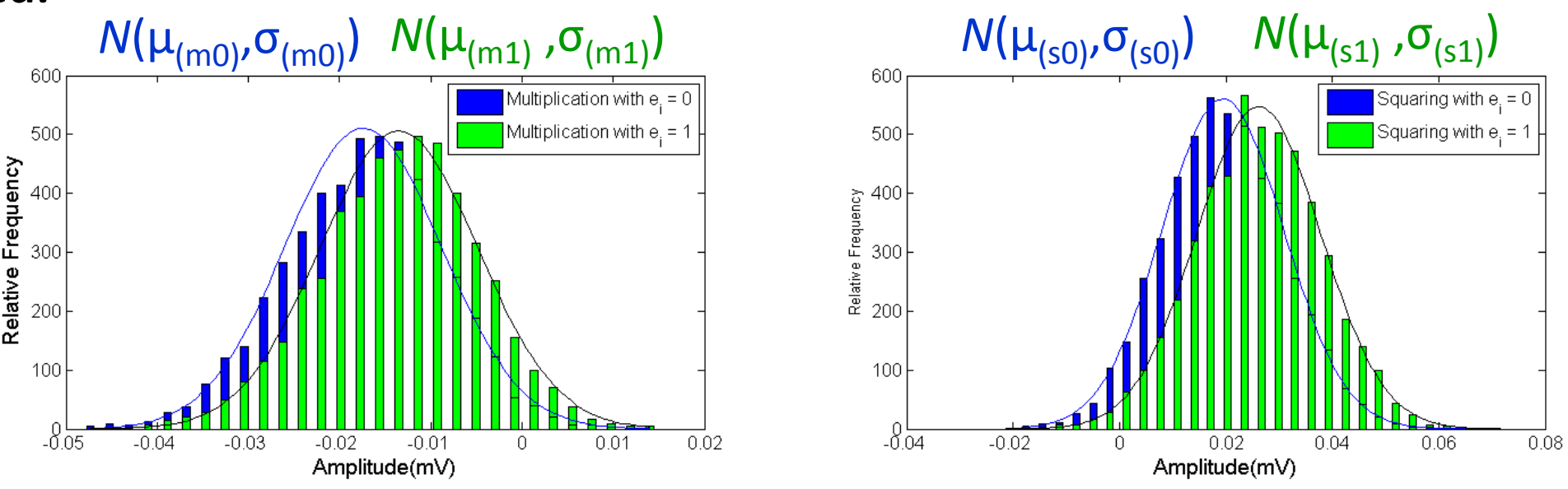

Protected:
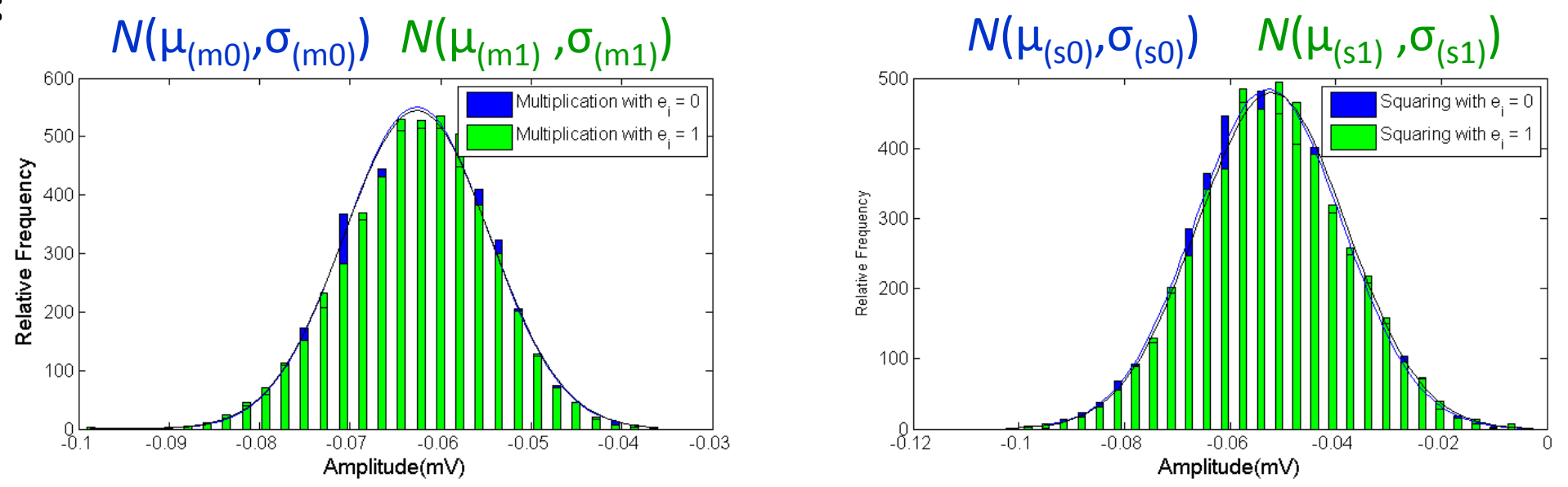


\section{Conclusions}

- We evaluated the combination of Algorithmic + Arithmetic + Hardware countermeasures against side-channel EM Analyses.

- LRA is a robust solution against simple, collisions, correlation and horizontal analyses (HW vs Trace).

- The major impact of LRA countermeasure is given in terms of memory (92\%), not time (1\%).

- Hardware countermeasures reduce the efficiency of single executions (trace) analysis on exponentiations (reduce the SNR).

\section{Future Works:}

- We will evaluate the effect of Algorithmic + Arithmetic + Hardware countermeasures against supervised and unsupervised template attacks. 
Thank you for your attention!

\section{QUESTIONS?}

perin@lirmm.fr 